\title{
Laser Welding of Vertically Aligned Carbon Nanotube Arrays on Polymer Workpieces
}

\author{
Jung Bin In ${ }^{\mathrm{a}, \star}$, Hyuk-Jun Kwon ${ }^{\mathrm{b}}$, Jae-Hyuck Yoo ${ }^{\mathrm{c}}$, Frances I. Allen ${ }^{\mathrm{d}, \mathrm{e}}$, Andrew M. Minor ${ }^{\mathrm{d}, \mathrm{e}}$, \\ Costas P. Grigoropoulos ${ }^{\mathrm{b}, *}$
}

\begin{abstract}
Here we demonstrate laser transmission welding of vertically aligned carbon nanotube (VACNT) arrays for joining polymer sheets. The unique characteristics of VACNTs make them suitable for use in laser welding. First, the excellent light absorption of the VACNTs induces selective heating at the contact plane with a polymer sheet, minimizing thermal damage to the polymer. Second, the porous and compliant structure of the VACNTs prevents the formation of air pockets inside the contact space. Successful welding is obtained when the laser irradiation power is at an optimal level, below which the adhesion is too weak and above which the excessive heat causes periodic damage along the scanning path. The optimized laser welding technique is expected to become a new method for implementing carbon nanotubes as mechanical linkers for various thermoplastic polymers.
\end{abstract}




\section{Introduction}

Laser processing has been steadily developed and widely applied in various fields of engineering owing to its unique advantages. For instance, as a non-contact process for easily deformable and sensitive structures, laser processing enables precise control of heat input and reduced thermal damage. Among its ubiquitous applications, laser light is employed in selective heating for welding a wide range of materials, including polymers, which are the key elements constituting daily commercial products.[1,2] The main process for laser welding is the absorption of the laser light to generate heat in polymers. The wavelength of the laser, the type of polymer, and the type and content of the pigment of the polymer affect the absorption and therefore should be controlled for effective laser welding. Laser welding techniques in standard use today can be divided into two main categories: laser butt welding and laser transmission welding. In laser butt welding, the joining surfaces are heated through a system of mirrors and then pressed together in the molten state. In laser transmission welding, which is adopted in this

study, an absorbing material is inserted between the joining surfaces and heated by absorbing a laser beam passing through the transparent workpiece.[1]

Recently, we developed a laser transmission-based welding technique to integrate vertically aligned carbon nanotube (VACNT) arrays and polymeric substrates into a functional composite.[3] VACNTs, which are also called carbon nanotube (CNT) forests, constitute a unique mesoporous material system comprising a self-assembled anisotropic network of CNTs. Individual CNTs feature an exceptionally high Young's modulus (ideally up to approximately 1 TPa) $[4,5]$ and tensile strength (11-63 GPa).[6] However, as a collective material system, VACNTs are mechanically compliant and exhibit foam-like behavior.[7] It has been revealed that the bundled and coiled structure of the nanotubes alleviates external loads.[8] That is, VACNTs behave like a porous cushion. By exploiting these unique characteristics, excellent contact between VACNTs and the polymer can be achieved, which is a crucial factor for successful welding.

In contrast to other heating techniques for CNT-substrate welding, such as hotplate and microwave heating,[9-11] the programmable patterning and transfer capability of the laser method enables the direct writing of VACNT patterns on thermoplastic polymer substrates such as polycarbonate (PC), polymethylmethacrylate, and polyethylene terephthalate. The heataffected zone induced by laser irradiation is relatively small, which minimizes thermal damage to 
the polymer. The heat can be selectively generated on the surface to be welded in a contact-free manner. Thus, the laser method allows the welding of complicated points and areas that are not readily accessible. Moreover, a designed weld geometry for obtaining sufficient mechanical strength can be achieved by scanning the welding laser.

While the transferred VACNTs could potentially be used as a flexible electrical element on the polymer sheets,[3] we found another promising use of this technique: the mechanical joining of polymers. In this study, the technique for laser welding VACNTs onto a polymer sheet is applied twice to join two polymer sheets. The VACNTs simultaneously serve as a light absorber[12] that converts the irradiating laser light into local heat for welding and as a linker material[13] that connects the two polymer sheets. The influence of the laser power on the strength of the weld is investigated systematically. The macro/micro morphologies of the VACNT-polymer interface welded using different laser powers are examined. In addition, the welding strength is measured in a tensile test to determine the optimal laser power for welding.

\section{Experimental}

\subsection{CVD synthesis of VACNTs}

For the catalytic chemical vapor deposition (CVD) growth of VACNTs, a catalyst film consisting of iron $(4 \mathrm{~nm}) /$ alumina $(30 \mathrm{~nm})$ was prepared on a silicon wafer by using electron beam evaporation and sputtering, respectively. VACNTs were produced in an atmospheric pressure CVD system. The catalyst substrate was annealed under a flow of hydrogen and argon gases to form nanoparticles, which are necessary templates for nucleation and growth of the CNTs. The growth was initiated by introducing ethylene at $740{ }^{\circ} \mathrm{C}$. To avoid the deposition of excessive amorphous carbon, the ethylene gas (200 standard cubic centimeters per minute (SCCM)) was diluted with argon (200 SCCM) and hydrogen (50 SCCM) during the growth period. Approximately $300 \mu \mathrm{m}$ of VACNTs were produced for $10 \mathrm{~min}$. Our previous work offers a more detailed description of the CVD procedure.[14]

\subsection{TEM characterization of VACNTs}

Portions of the CNT arrays were scratched from the substrates and deposited onto copper grids for inspection by transmission electron microscopy (TEM) using a 200MC Zeiss Libra transmission electron microscope operated at $200 \mathrm{kV}$. Surveys of the CNT diameters and the number of carbon layers making up the multi-walled nanotubes were performed by acquiring 
high-resolution TEM images of 40 CNTs and analyzing them using DigitalMicrograph ${ }^{\mathrm{TM}}$ software. Scanning electron microscopy (SEM) of CNTs was conducted using a FEI Nova NanoSEM 650 scanning electron microscope.

\subsection{Laser welding setup}

A galvanometer mirror system (SCANLAB hurrySCAN II-14) was coupled to a continuous-wave argon ion laser (wavelength: $514 \mathrm{~nm}$; Lexel 3000) to direct the laser beam onto the VACNT-PC interface. This setup enabled dynamic scanning with minimal change of the scanning speed. The beam spot size was estimated to be $50 \mu \mathrm{m}$. The scanning speed was fixed at $1 \mathrm{~mm} / \mathrm{s}$. For areal patterning, contour scanning was conducted with a $20-\mu \mathrm{m}$ scan spacing to ensure complete welding of the CNTs over the entire pattern area.

\subsection{Tensile test}

The tensile strength of the weld was measured using a home-built measurement system consisting of a motorized linear stage and a force sensor (Dillon GL025). An epoxy adhesive (Loctite ${ }^{\circledR}$ ) was coated on the opposite side of the laser-welded VACNT-PC sample by using a doctor-blade method. As soon as the VACNTs came into contact with the epoxy layer (detected by the force sensor), the contact region was illuminated with bright halogen light through a small hole for complete and fast curing. The intensity of the illumination was adjusted to avoid excessive heating of the PC-VACNT-epoxy weld, and the temperature was monitored using a non-contact thermometer (Micro-Epsilon CT-SF22).

\section{Results and Discussion}

\subsection{Laser transmission welding of VACNTs and polycarbonate}

Fig. 1 shows a schematic of the laser transmission welding process used. The basic welding method is consistent with our previously reported technique.[3] CNTs are thermally stable at the melting temperatures of most polymers. Thus, the entire welding process was conducted under ambient conditions. The as-grown VACNT sample was covered with the polymer substrate and a glass slide. The glass slide was pressed down using mechanical clamps with spacers to enhance the VACNT-polymer contact. Then, the first welding step was performed by irradiating the top of the VACNTs with an $\mathrm{Ar}^{+} \mathrm{CW}$ laser beam (wavelength: 514 $\mathrm{nm}$ ) through the glass-polymer stack. In laser transmission welding, the purpose of the laser 
irradiation is to heat the weld interface selectively, and direct absorption of the incident light by the polymer should be avoided. Thus, transparent PC sheets were used as the polymer substrate. Instead, the VACNTs, which are known as an extremely dark material,[12] absorbed most of the laser light, increasing the temperature of the contact surface of the PC sheet above its glass transition temperature. After the completion of the laser scanning and the separation of the welded PC sheet from the as-grown VACNTs, the first VACNT-polymer weld was obtained. To fabricate a welding joint of two polymer sheets, the first weld was used as a VACNT support. The same welding process was repeated with the second polymer sheet.

Different kinds of polymers can be used as the second sheet, provided that they are compatible with the laser welding technique. For instance, a PC-VACNT-acrylic plate assembly was fabricated using an acrylic plate as the second sheet (Fig. 1b). As shown in Fig. 1c, the welded area was bonded so tightly that no immediate leakage of green fluorescent liquid (polymer microspheres, $1 \%$ solid; Duke Scientific) was observed. The empty inner pocket of the welding pattern was filled with the colloid via a syringe needle. It should be noted that VACNTs constitute a mesoporous material system with a spacing of 10-100 nm between the nanotubes. Therefore, despite the tight adhesion, a liquid can eventually infiltrate the welded VACNTs. A welded assembly $\left(1 \mathrm{~cm}^{2}\right.$ in area) was able to withstand an external load (approximately 3.79 liters (one gallon) of water) for longer than 10 minutes, as shown in Fig. 1d. The welded PC sheets and the binder clip were fixed using an epoxy adhesive.

(a)
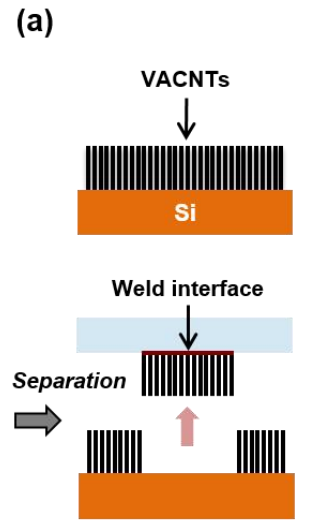
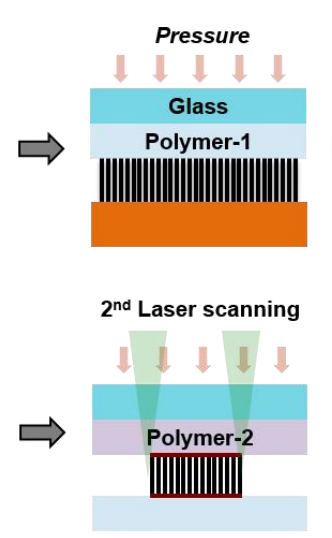

$2^{\text {nd }}$ Laser scanning

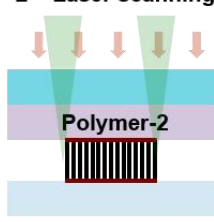

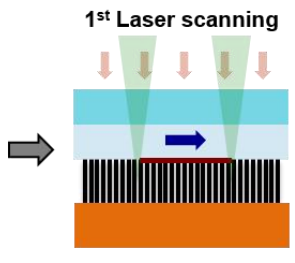

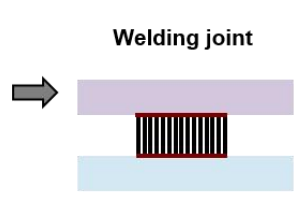

(b)

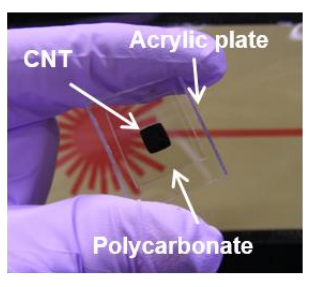

(c) Tight bond

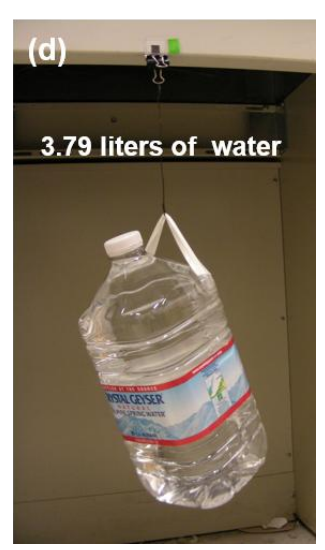

Fig. 1. (a) Schematic of the laser transmission welding process. (b) Photograph of a laser-welded PCVACNT-acrylic plate assembly. (c) Photograph of a leak test of a PC-VACNT-PC assembly. (d) 3.79 liters (one gallon) of water hanging onto a PC-VACNT-PC weld. 


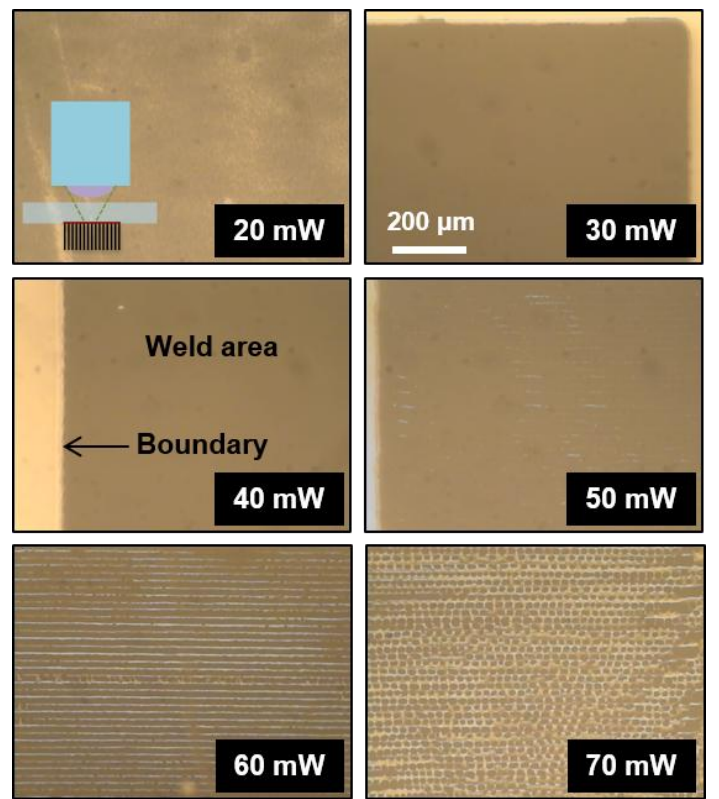

Fig. 2. Optical microscopy of the VACNTPC interface welded using different laser powers $(20-70 \mathrm{~mW})$. The scanning direction is horizontal. The inset diagram shows the imaging configuration.
Similar to the conventional laser transmission welding of plastics, the laser welding of VACNTs and thermoplastic polymer materials involves (1) heating by the absorption of the laser light, (2) intermixing of the CNT extremities and the contacting polymer at a temperature above the glass transition point of the polymer, (3) heat dissipation in the wake of the scanning laser beam, and (4) solidification of the polymer and completion of the welding. The photothermal process that leads to the polymer phase change and increase in adhesion is expected to depend on the laser light intensity. Moreover, it is intuitively expected that there exists a laser power range below which the adhesion is insufficient and above which excessive heating generates thermal damage in the polymer.

To elucidate the effect of the laser power on the VACNT-PC weld, various laser-welding powers were examined, and the resulting strength of the welds was characterized.

\subsection{Influence of laser power}

A series of laser-welded VACNT-PC samples were prepared by increasing the laser power from 20 to $70 \mathrm{~mW}\left(1.0-3.6 \mathrm{~kW} / \mathrm{cm}^{2}\right)$ in increments of $10 \mathrm{~mW}$. The welding threshold was discovered to be $15 \mathrm{~mW}$ (see supplementary materials); higher powers were applied to achieve reproducible welding. Raster scanning was conducted following a serpentine path to weld a square area $\left(2 \times 2 \mathrm{~mm}^{2}\right)$. Fig. 2 shows optical microscopy images of the VACNT-PC interface welded at the various laser powers. The bright-field images were captured through the transparent polymer sheet as shown in the inset diagram. At a laser power of $20 \mathrm{~mW}$, the texture of the interface was visible, whereas the interface appeared uniformly dark with no sign of air pockets from the samples prepared at higher powers $(30,40 \mathrm{~mW})$. However, at $50 \mathrm{~mW}$, a defective interface started to appear along the laser-scanning path, which became increasingly visible at higher powers. 
The tensile strength of the prepared weld samples was measured using a custom-built force-measurement system (see the Experimental section). Fig. 3a describes the measurement procedure. A welded VACNT-PC sample was backed with a glass slide and attached to the sensor head of the measurement device, which was mounted on a motorized stage. Epoxy glue was applied to the opposite fixed wall using a doctor-blade method. The stage was moved forward so that the tips of the VACNTs touched the epoxy, and the epoxy was fully cured. The stage was then moved back at a speed of $0.12 \mathrm{~mm} / \mathrm{min}$ to apply tensile force until failure of the welded structure occurred. As shown in Fig. 3b, the failure left CNTs on both sides (albeit less in the case of the 20-mW weld side), indicating fracture of the CNTs during the tensile test, which suggests that the laser-induced adhesion strength of the VACNT-PC weld was greater than the tensile strength of the VACNTs.
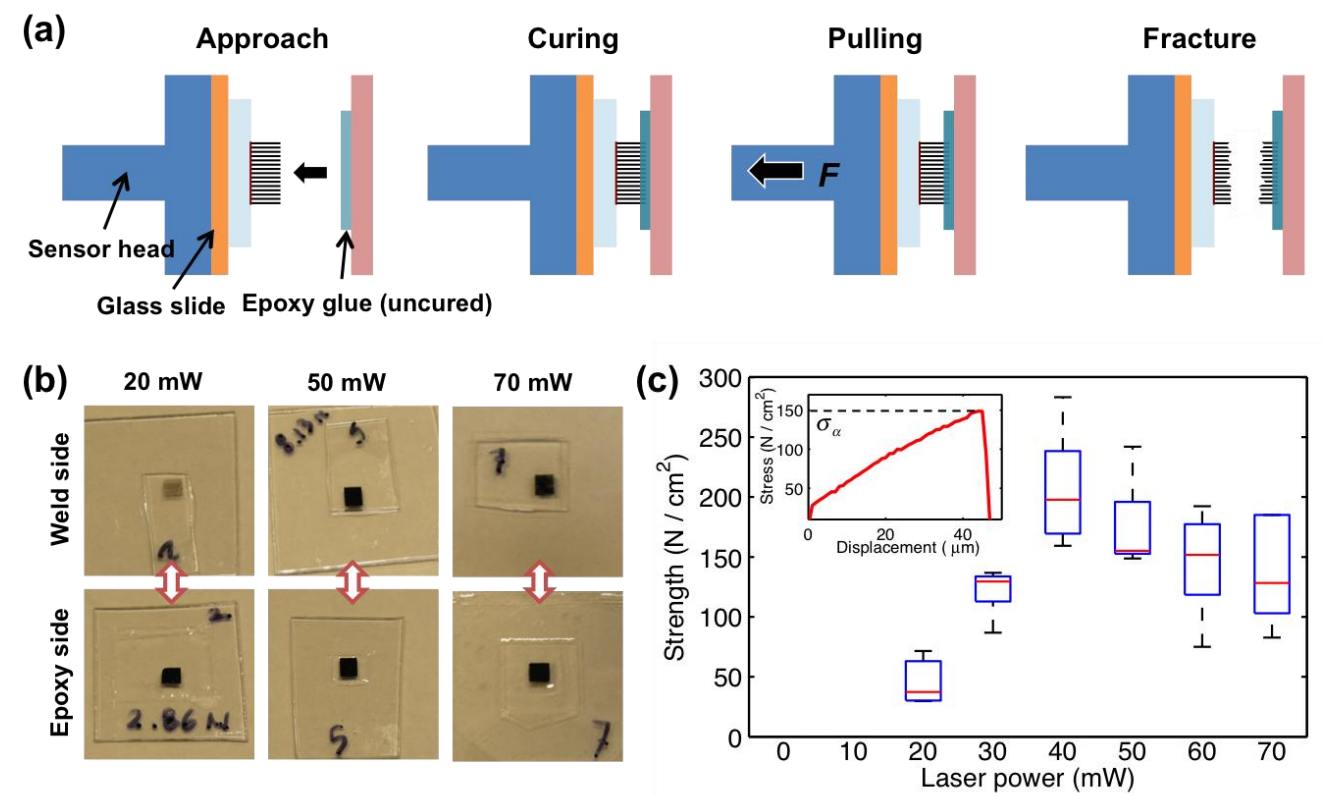

Fig. 3. (a) Schematic of the force measurement. (b) Photographs of the separated pieces of laser-welded and epoxy-applied PC-VACNT test samples after the tensile test. (c) Plot of the weld strength versus the laser-welding power. The inset shows a representative effective stress-displacement curve obtained from a 50-mW welded sample. $\sigma_{\alpha}$ indicates the tensile strength of the as determined in this study.

Fig. 3c shows the relationship between the measured tensile strength and the welding laser power. Five samples were tested for each laser power. The strength increased as the laser power increased from 20 to $40 \mathrm{~mW}$, but at and above $50 \mathrm{~mW}$, it showed a downward trend. The 
maximum strength of $280 \mathrm{~N} / \mathrm{cm}^{2}(2.8 \mathrm{MPa})$ was obtained for the laser power of $40 \mathrm{~mW}$. The inset in Fig. 3c shows a representative stress-displacement plot obtained for a VACNT-PC sample welded at $50 \mathrm{~mW}$.

To estimate the tensile strength of the individual CNTs, dimensional information on the CNTs is necessary. Transmission electron microscopy (TEM) revealed that the average outer diameter was approximately $15 \mathrm{~nm}$ and the average number of walls of the CNTs were approximately 12 (Fig. 4). The number density was calculated to be $4.5 \times 10^{9} \mathrm{CNTs} / \mathrm{cm}^{2}$. Finally, the tensile strength of the individual CNTs was estimated to be approximately $0.35 \mathrm{GPa}$. This is significantly lower than previously reported strengths of individual CNTs (11-63 GPa) measured for single CNT samples.[6]

This large discrepancy in the tensile strength of the individual CNTs could be attributed to (1) inherent structural defects of the nanotubes (see Fig. S2 in supplementary materials), (2) the uneven distribution of the external load on the nanotubes, and (3) the density variation along the length of the VACNTs.[15] It is known that VACNTs become wavy due to Van der Waals interaction with the neighboring nanotubes when grown in a low density and due to defects in the crystalline structure.[15-17] However, at the same time, some nanotubes grow straight despite the neighboring wavy nanotubes. When an external load is applied, the tensile force is preferentially transferred to the straight CNTs, while the tortuous structure of the other CNTs mitigate the force since they can stretch farther than the straight nanotubes.[8] Moreover, the number density of the VACNTs varies along the longitudinal direction because of the evolutionary growth mechanism and the different lifetimes of the catalytic growth seeds.[16] For these reasons, only a few of the nanotubes are subject to the
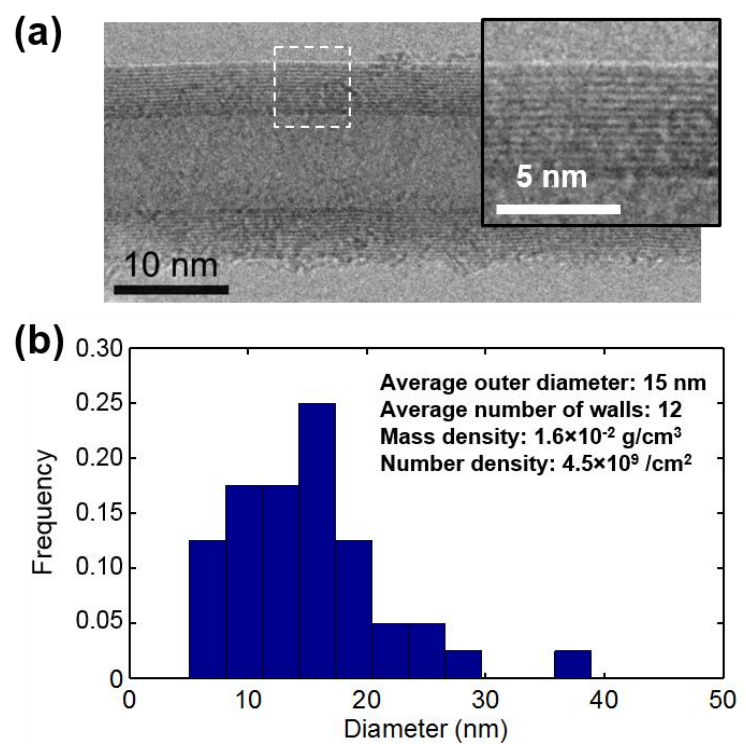

Fig. 4. (a) TEM image of a representative multi-walled CNT from the VACNTs used in this study. The inset shows a magnified view of the area marked. (b) Diameter distribution of the multi-walled CNTs. The measured average diameter, average number of walls, mass density, and number density of the CNTs are given. 
tensile force, resulting in a significantly reduced strength of the VACNTs.

To demonstrate this concept, SEM of the as-grown VACNTs (Fig. 5a) revealed that only a few of the VACNTs were straight and thereby accommodating the brunt of the tensile force. Side-view SEM (Fig. 5b and c) of the VACNTs after the tensile test revealed relatively straight nanotubes on the very top of the fracture plane. Their number density was far lower than that of the as-grown CNTs (Fig. 5a). The fracture plane was found to be irregular at the microscopic scale, although it appeared flat over the large scale.
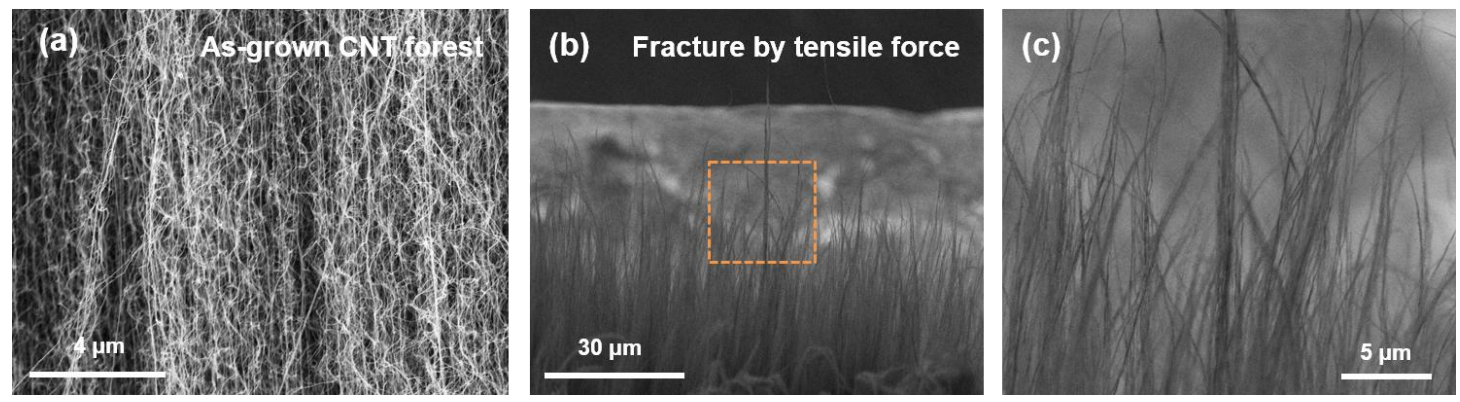

Fig. 5. (a) SEM (side view) of the middle part of the as-grown VACNTs, showing two types of CNTs: straight and tortuous. (b) SEM (side view) of the VACNTs (epoxy side) after fracture in the tensile test. (c) Enlarged view of the marked area in (b).

To better understand the effect of laser power on the weld strength, the morphology of the fractured VACNTs after the tensile test was inspected using optical microscopy and SEM (Fig. 6a). In the case of the 20-mW weld, short CNTs were left on the weld surface as a result of fracture. Fig. $6 \mathrm{~b}$ is an SEM image of the cross section of an as-prepared 20-mW weld. In the image, the short remnant nanotubes are identified as the nanotubes pinned to the polymer surface by welding. The image reveals that only a few of the nanotubes adhered to the polymer surface. Obviously, the welding power is closely related to the number of pinned nanotubes and therefore the strength of the weld. Thus, it can be argued that the $20-\mathrm{mW}$ power was insufficient for the complete welding of the VACNTs and the PC sheet. At higher powers, more complete adhesion was achieved at the weld interface (Fig. 6c). These results also support the trend observed in Fig. $3 \mathrm{c}$.

Considering that the temperature of the PC surface should at least reach the glass transition temperature (approximately $150^{\circ} \mathrm{C}$ ) at the beginning of the welding (approximately 15 $\mathrm{mW}$ ), it is reasonably expected that not only significant melting (melting point: approximately $230^{\circ} \mathrm{C}$ ) but also vaporization of the PC occurred at the high powers examined. Thus, the increase 
in the strength of the weld at increased power is attributed to the possible enhanced wetting of the CNTs by the polymer melt driven by swelling and capillary force. Excessive power (i.e., 70 $\mathrm{mW}$ ) caused defects at the weld interface, as shown on the right of Fig. 6a. Fig. 6d shows an SEM image of the $70 \mathrm{~mW}$-welded CNTs after fracture. Together with the spotted damage observed in the microscopy image (Fig. 2), the circular holes in Fig. 6d suggest that the separation was partly initiated at the damaged weld interface. Fig. 6e, which provides a magnified view of the marked area in Fig. 6d, shows the material (PC) that is presumably vaporized and condensed on the sidewalls of the CNTs. Hence we propose that significant vaporization of PC at the interface and the subsequent loss of contact surface were possible causes of the decrease in the measured weld strength at higher powers.

(a)

$20 \mathrm{~mW}$
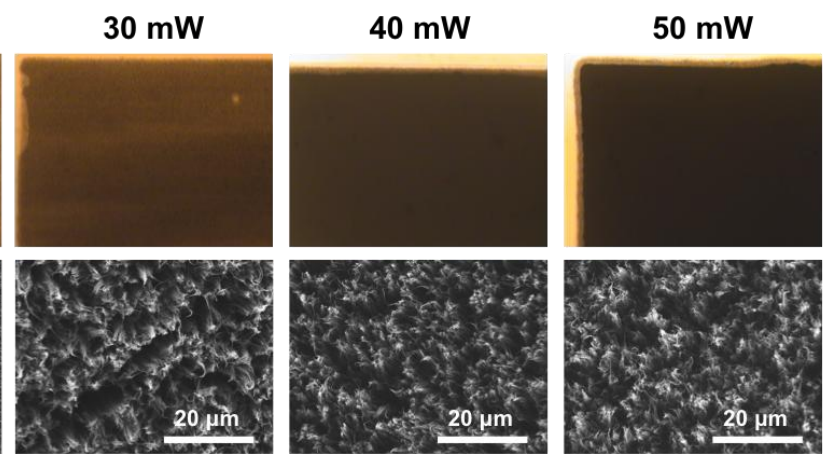

$70 \mathrm{~mW}$

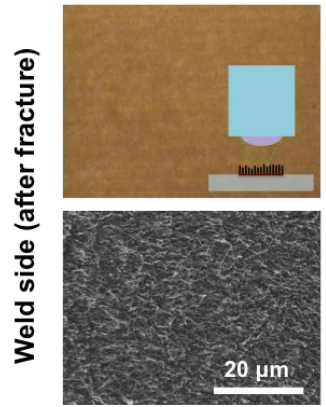

(b)
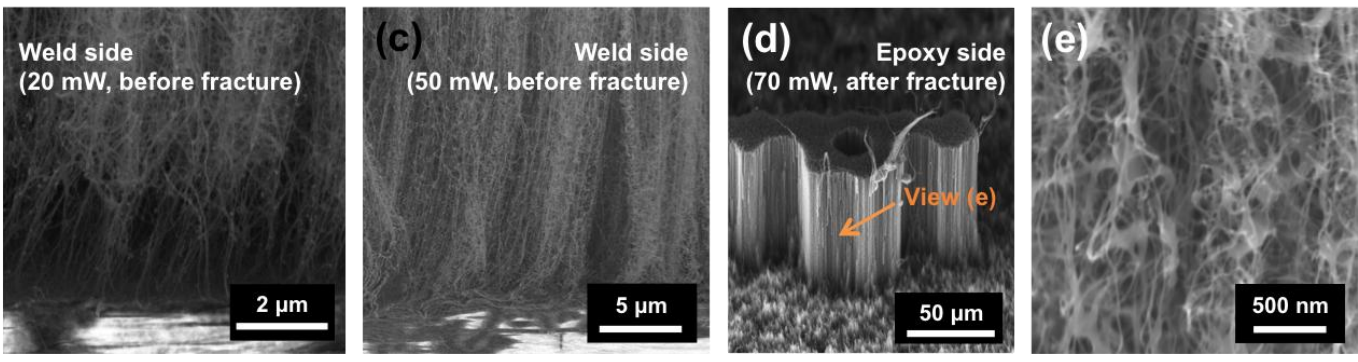

Fig. 6. (a) Series of optical microscopy images (bright-field, top view) and corresponding SEM images (top view) of the CNTs on the weld side after fracture in the tensile test. The inset schematic describes the configuration of the optical microscopy imaging. For the $70-\mathrm{mW}$ welded sample, the SEM image was captured for a CNT-covered spot, as indicated in the optical microscopy image. (b) SEM image (side view) of laser-welded CNT-PC $(20 \mathrm{~mW})$ before the tensile test. The thin PC sheet was manually torn to expose the weld interface. (c) SEM image (side view) of laser-welded CNT-PC (50 mW) obtained in the same way. (d) SEM image ( $45^{\circ}$ tilt view) of a $70-\mathrm{mW}$ welded sample (epoxy side) after fracture in the tensile test. (e) Enlarged SEM view for the location marked in (d). 


\subsection{Potential uses of laser transmission welding of VACNTs}

In this study, VACNTs served as a porous and adaptable cushion, enabling excellent contact with polymer workpieces and preventing the formation of air pockets during welding. The laser welding technique can be used for joining flexible polymer substrates into compliant structures. The polymer-VACNT-polymer sandwich structure can for example be applied in flexible light-shielding VACNT applications.[12, 18] This unique structure can also be used as a mechanical framework for the fabrication of VACNT composites. For instance, Kim et al. demonstrated the fabrication of a VACNT-polymer composite by infiltrating a polymer solution into the porous VACNTs of the sandwich structure. Disturbance of the CNT orientation during the solution processing was successfully avoided since both ends of the nanotubes were mechanically supported.[19]

Despite the applicability of VACNTs as a linker material for laser welding, the measured strength of our VACNTs was relatively weak compared to standard weld strengths. As discussed, the relative weakness of the weld assembly was caused by insufficient strength of the VACNTs themselves, and not the actual welded interface. However, by optimizing the growth parameters to produce high-density VACNTs [20] with greatly enhanced strength, we envision superior VACNT linkers for laser welding in the future.

\section{Conclusions}

Laser transmission welding of VACNTs and polymeric substrates was demonstrated. The features of this technique include (1) applicability in ambient conditions, (2) dry and instant processes, (3) programmable weld patterning, and (4) minimal thermal damage to the workpieces. The tensile strength of the VACNT-PC weld increased with increasing laser power, but the use of an excessively high welding power undermined the strength. The adhesion strength at the weld interface achieved by laser welding using an optimized laser power was greater than the tensile strength of the VACNTs tested in this work. Therefore, we conclude that the weld strength is determined by the laser intensity used in the welding process and ultimately by the intrinsic strength of the VACNTs. 


\section{Acknowledgements}

This work was supported by Basic Science Research Program (No. NRF2015R1C1A1A01052523) and Nano·Material Technology Development Program (No. NRF2016M3A7B4910532) through the National Research Foundation of Korea (NRF) grant funded by the Ministry of Science, ICT and Future Planning. TEM analysis was performed at the Molecular Foundry, which is supported by the Office of Science, Office of Basic Energy Sciences, for the U.S. Department of Energy under Contract No. DE-AC02-05CH11231. Lawrence Livermore National Laboratory is operated by Lawrence Livermore National Security, LLC, for the U.S. Department of Energy, National Nuclear Security Administration under Contract DE-AC52-07NA27344. J.I. thanks Prof. Seok-Min Kim (Chung-Ang University) for the use of RAMAN spectroscopy.

\section{References}

[1] H. Potente, O. Karger, G. Fiegler, Laser and microwave welding - The applicability of new process principles, Macromol Mater Eng 287(11) (2002) 734-744.

[2] P. Mapleston, Plastics welding: The choices widen, Plast Eng 64(4) (2008) 10-+.

[3] J.B. In, D. Lee, F. Fornasiero, A. Noy, C.P. Grigoropoulos, Laser-Assisted Simultaneous Transfer and Patterning of Vertically Aligned Carbon Nanotube Arrays on Polymer Substrates for Flexible Devices, ACS nano 6(9) (2012) 7858-7866.

[4] M.M.J. Treacy, T.W. Ebbesen, J.M. Gibson, Exceptionally high Young's modulus observed for individual carbon nanotubes, Nature 381(6584) (1996) 678-680.

[5] E.W. Wong, P.E. Sheehan, C.M. Lieber, Nanobeam mechanics: Elasticity, strength, and toughness of nanorods and nanotubes, Science 277(5334) (1997) 1971-1975.

[6] M.F. Yu, O. Lourie, M.J. Dyer, K. Moloni, T.F. Kelly, R.S. Ruoff, Strength and breaking mechanism of multiwalled carbon nanotubes under tensile load, Science 287(5453) (2000) 637-640.

[7] M. Xu, D.N. Futaba, T. Yamada, M. Yumura, K. Hata, Carbon Nanotubes with Temperature-Invariant Viscoelasticity from-196 to 1000 C, Science 330(6009) (2010) 1364-1368.

[8] N.J. Ginga, W. Chen, S.K. Sitaraman, Waviness reduces effective modulus of carbon nanotube forests by several orders of magnitude, Carbon 66 (2014) 57-66.

[9] C. Wang, T. Chen, S. Chang, S. Cheng, T. Chin, Strong Carbon-Nanotube-Polymer Bonding by Microwave Irradiation, Advanced Functional Materials 17(12) (2007) 1979-1983.

[10] H.C. Shim, Y.K. Kwak, C.-S. Han, S. Kim, Enhancement of adhesion between carbon nanotubes and polymer substrates using microwave irradiation, Scripta Materialia 61(1) (2009) 32-35.

[11] T.Y. Tsai, C.Y. Lee, N.H. Tai, W.H. Tuan, Transfer of patterned vertically aligned carbon nanotubes onto plastic substrates for flexible electronics and field emission devices, Applied Physics Letters 95(1) (2009) 013107.

[12] Z.P. Yang, L.J. Ci, J.A. Bur, S.Y. Lin, P.M. Ajayan, Experimental observation of an extremely dark material made by a low-density nanotube array, Nano Letters 8(2) (2008) 446-451. 
[13] X. Jin, J. Strueben, L. Heepe, A. Kovalev, Y.K. Mishra, R. Adelung, et al., Joining the Un-Joinable: Adhesion Between Low Surface Energy Polymers Using Tetrapodal ZnO Linkers, Advanced Materials 24 (2012) 5676-5680.

[14] J.B. In, C.P. Grigoropoulos, A.A. Chernov, A. Noy, Growth Kinetics of Vertically Aligned Carbon Nanotube Arrays in Clean Oxygen-free Conditions, ACS nano 5(12) (2011) 9602-9610.

[15] F.A. Hill, T.F. Havel, A.J. Hart, C. Livermore, Enhancing the tensile properties of continuous millimeter-scale carbon nanotube fibers by densification, ACS applied materials \& interfaces 5(15) (2013) 7198-7207.

[16] M. Bedewy, E.R. Meshot, H. Guo, E.A. Verploegen, W. Lu, A.J. Hart, Collective Mechanism for the Evolution and Self-Termination of Vertically Aligned Carbon Nanotube Growth, Journal of Physical Chemistry C 113(48) (2009) 20576-20582.

[17] M.R. Maschmann, Integrated simulation of active carbon nanotube forest growth and mechanical compression, Carbon 86 (2015) 26-37.

[18] N. Li, Y. Huang, F. Du, X.B. He, X. Lin, H.J. Gao, et al., Electromagnetic interference (EMI) shielding of single-walled carbon nanotube epoxy composites, Nano Letters 6(6) (2006) 1141-1145.

[19] S. Kim, F. Fornasiero, H.G. Park, J. Bin In, E. Meshot, G. Giraldo, et al., Fabrication of flexible, aligned carbon nanotube/polymer composite membranes by in-situ polymerization, J Membrane Sci 460 (2014) 91-98.

[20] G. Zhong, J.H. Warner, M. Fouquet, A.W. Robertson, B.A. Chen, J. Robertson, Growth of Ultrahigh Density Single-Walled Carbon Nanotube Forests by Improved Catalyst Design, ACS nano 6(4) (2012) 2893-2903. 

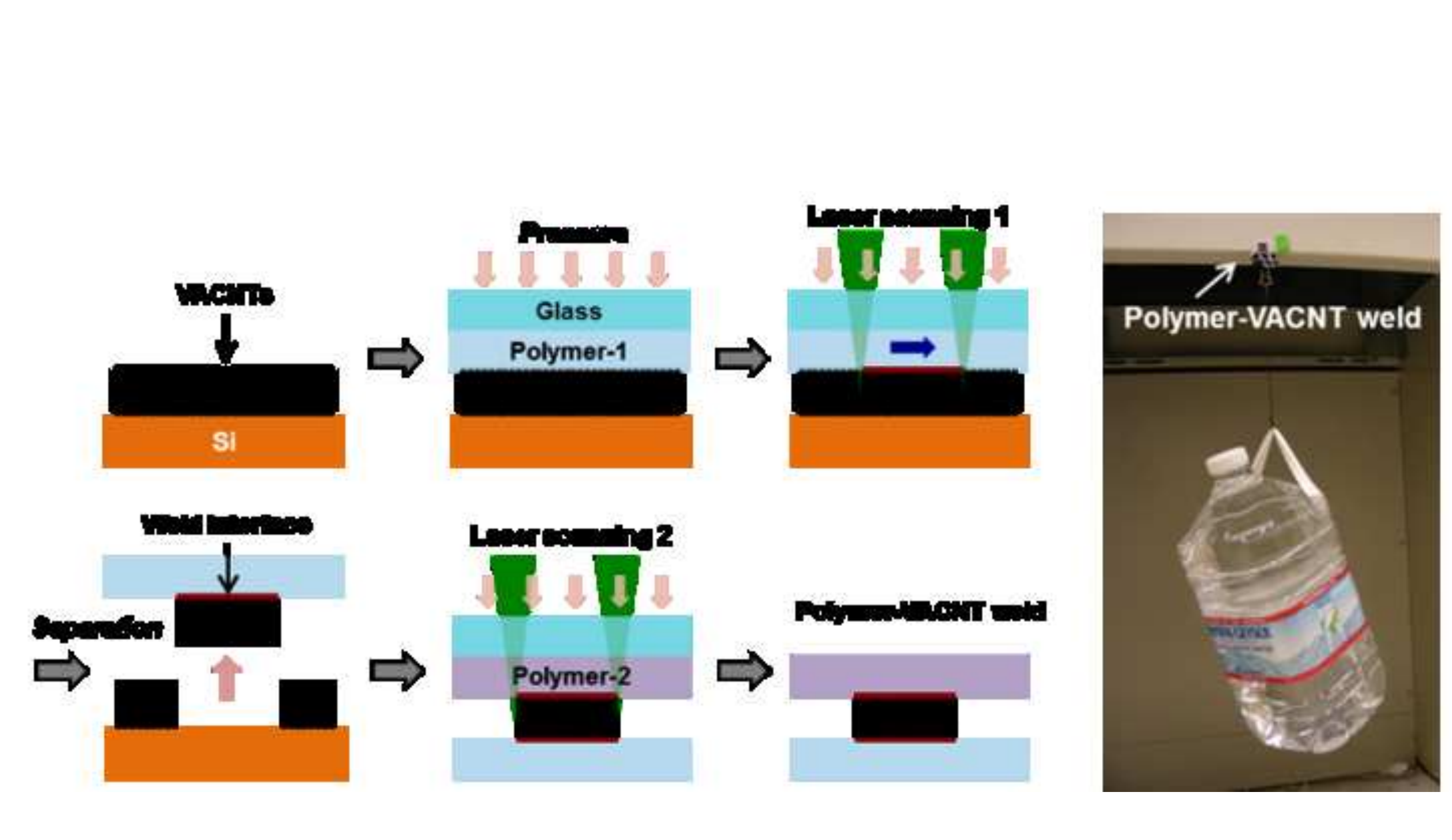